\title{
„Der Ausschluss von Arzneimitteln in der gesetzlichen Krankenversicherung Zu Inhalt und Reichweite des § 34 SGB V““
}

Dissertation vorgelegt von Dorothea Dettling

Erstgutachter: Prof. Dr. Peter Axer

Zweitgutachter: Prof. Dr. Bernd Grzeszick

Institut für deutsches und europäisches Verwaltungsrecht 
Arzneimittel sind für die Krankenbehandlung und die Sicherstellung einer hochwertigen medizinischen Versorgung unverzichtbar, stellen jedoch ebenso, neben der Krankenhausbehandlung und der ärztlichen Behandlung, einen der größten Ausgabenposten innerhalb der gesetzlichen Krankenversicherung dar. Um diese hohen Kosten einzudämmen, ist der Gesetzgeber gehalten Kostenregulierungsinstrumente einzuführen, wozu auch Arzneimittelausschlüsse gehören. Die Arbeit untersucht die Ausschlüsse von Arzneimitteln in der gesetzlichen Krankenversicherung, wobei sich die Untersuchung auf § 34 SGB V beschränkt.

\section{A. Arzneimittelversorgung in der gesetzlichen Krankenversicherung}

Versicherte haben nach $\S 27$ Abs. 1 S. 1 SGB V Anspruch auf Krankenbehandlung, zu der auch die Versorgung mit Arzneimitteln gehört, wenn die Krankenbehandlung notwendig ist, um eine Krankheit zu erkennen, zu heilen, ihre Verschlimmerung zu verhüten oder Krankheitsbeschwerden zu lindern. Grundvoraussetzung für einen Anspruch auf Versorgung mit Arzneimitteln ist somit das Vorliegen von Krankheit, mithin der Eintritt des Versicherungsfalles. Der Anspruch auf Versorgung mit Arzneimitteln stellt sich dabei grundsätzlich als Sachleistungsanspruch dar. Erforderlich für einen Anspruch auf Versorgung mit Arzneimitteln nach $\S 31$ SGB V ist zudem, dass das Arzneimittel zugelassen und damit verkehrsfähig ist. Allein aus der Zulassung resultiert aber noch kein Versorgungsanspruch des Versicherten. Die Zulassung ist negativ vorgreiflich und stellt lediglich eine Mindestvoraussetzung dar. Sie begründet aber nicht bereits den Leistungsanspruch des Versicherten.

Der nach $\S 27$ Abs. 1 SGB V bestehende Anspruch auf Versorgung mit Arzneimitteln bei Vorliegen einer Krankheit wird durch zahlreiche Regelungen im Leistungsrecht in den $\S \S 31 \mathrm{ff}$. SGB V sowie im Leistungserbringungsrecht in den $\S \S 129 \mathrm{ff}$. SGB V und durch die Arzneimittel-Richtlinie des Gemeinsamen Bundesausschusses gem. § 92 Abs. 1 S. 1, 2 Nr. 6 SGB V begrenzt. Der Anspruch des Versicherten auf Versorgung mit Arzneimitteln wird demnach durch verschiedene gesetzliche und untergesetzliche Regelungen beschränkt, wozu etwa der in $\S 31$ Abs. 1 S. 1 SGB V geregelte Ausschluss apothekenpflichtiger Arzneimittel, die in $\S 34$ SGB V geregelten Ausschlüsse von nicht verschreibungspflichtigen Arzneimitteln, Bagatellarzneimitteln, Lifestyle-Arzneimitteln und unwirtschaftlichen Arzneimitteln, die Verordnungsausschlüsse und Verordnungseinschränkungen durch die Arzneimittel-Richtlinie sowie die Zuzahlungsregelung des $\S 31$ Abs. 3 SGB V, die Festbetragsregelung des $\S 35$ SGB V, die frühe Nutzen-Bewertung und die Kosten-Nutzen-Bewertung in $\S \S 35 \mathrm{a}, 35 \mathrm{~b}$ SGB V oder die die Aut-idem-Substitution in $§ 129$ Abs. 1 S. 1 Nr. 1 SGB V zählen.

\section{B. Der Ausschluss nicht verschreibungspflichtiger Arzneimittel - §34 Abs. 1 S. 1-5 SGB V}

Der gesetzliche Ausschluss nicht verschreibungspflichtiger Arzneimittel nach § 34 Abs. 1 S. 1 SGB V schließt eine ganze Produktkategorie von Arzneimitteln von der Versorgung der gesetzlichen Krankenversicherung aus. Bei den nicht verschreibungspflichtigen Arzneimitteln, die auch als OTC-Arzneimittel bezeichnet werden, wobei OTC für „over the counter“ steht, handelt es sich um Arzneimittel, die ohne ein ärztliches Rezept in der Apotheke abgegeben werden und die damit frei über die Ladentheke verkauft werden können. $\mathrm{Zu}$ den nicht verschreibungspflichtigen Arzneimitteln zählen - in Abgrenzung zu verschreibungspflichtigen Arzneimitteln - solche, die nur geringe Nebenwirkungen haben, durch die keine unmittelbare oder mittelbare Gefährdung der Gesundheit besteht und deren Wirkstoffe wissenschaftlich bekannt sind. Dabei handelt es sich aber, anders als der Ausschluss suggerieren mag, nicht um entbehrliche Arzneimittel, deren Einnahme medizinisch nicht notwendig ist. Angeknüpft hat 
der Gesetzgeber mit der Verschreibungspflicht an ein Kriterium des Arzneimittelrechts, das mithin ein Kriterium der Arzneimittelsicherheit darstellt.

Zur Abmilderung des Ausschlusses hat der Gesetzgeber zwei Ausnahmen im Gesetz geregelt: zum einen die Verordnungsfähigkeit nicht verschreibungspflichtiger Arzneimittel bei Kindern bis zur Vollendung deren 12. Lebensjahres oder bei Jugendlichen bis zur Vollendung deren 18. Lebensjahres, sofern diese unter Entwicklungsstörungen leiden, zum anderen die Verordnungsfähigkeit nicht verschreibungspflichtiger Arzneimittel bei Vorliegen einer schwerwiegenden Erkrankung, sofern sich das Arzneimittel als Standardtherapeutikum zur Behandlung einer schwerwiegenden Erkrankung darstellt, wobei der Gesetzgeber die Festlegung, welche Arzneimittel dieser Ausnahme unterfallen, dem Gemeinsamen Bundesausschuss übertragen hat. Der Gemeinsame Bundesausschuss hat dementsprechend eine Liste, die sog. OTCÜbersicht, erstellt, auf der sich bestimmte schwerwiegende Erkrankungen sowie der dazugehörige Standardwirkstoff zur Behandlung finden.

Des Weiteren hat der Gemeinsame Bundesausschuss die Begriffe der schwerwiegenden Erkrankung und des Therapiestandards definiert. Dabei hat der Gemeinsame Bundesausschuss den Begriff der schwerwiegenden Erkrankung an den Begriff der schwerwiegenden Erkrankung im Off-Label-Use angelehnt. Von den Gerichten wird dieser Begriff daher ebenso wie beim Off-Label-Use streng und restriktiv ausgelegt, was es im Rahmen der OTC-Liste aufgrund der unterschiedlichen Gefahrenlage zum Off-Label-Use und unter Berücksichtigung der Beispiele in der Gesetzesbegründung, in der etwa das Klimakterium genannt wird, nicht bedarf. Es erscheint zwar sachgerecht den Begriff der schwerwiegenden Erkrankung ebenso wie beim Off-Label-Use als eine lebensbedrohliche oder die Lebensqualität auf Dauer nachhaltig beeinträchtigende Erkrankung zu definieren, allerdings ist die zweite Alternative der lebensbeeinträchtigenden Erkrankung weniger streng als beim Off-Label-Use auszulegen, sodass nicht nur Erkrankungen, die sich in ihrer Schwere und Seltenheit vom Durchschnitt der Erkrankungen abheben, dem Begriff unterfallen. Erkrankungen, die die Lebensqualität auf Dauer beeinträchtigten, können im Rahmen von $\S 34$ Abs. 1 S. 2 SGB V ebenso solche Erkrankungen sein, die zu erheblichen Beeinträchtigungen führen, sodass der Alltag in gewohnter Weise nicht mehr bewerkstelligt werden kann, und die eine Schwere derart aufweisen, dass ein unabweisbarer Bedarf an einer Versorgung mit Arzneimitteln besteht.

Die Definition des Therapiestandards durch den Gemeinsamen Bundesausschuss, wonach ein Arzneimittel dann als Therapiestandard gilt, wenn der therapeutische Nutzen zur Behandlung der schwerwiegenden Erkrankung dem allgemein anerkannten Stand der medizinischen Erkenntnisse entspricht, ist allerdings sachgerecht. Daneben besteht für die Krankenkassen die Möglichkeit nicht verschreibungspflichtige Arzneimittel als Satzungsleistung zu gewähren und dadurch den Ausschluss abzumildern.

\section{Verfassungsmäßigkeit und Europarechtskonformität des Ausschlusses nicht ver- schreibungspflichtiger Arzneimittel}

Durch Leistungsausschlüsse werden pharmazeutische Unternehmer in ihren Absatzmöglichkeiten eingeschränkt, Ärzte in ihrem Verordnungsverhalten beschränkt und den Versicherten werden Leistungen der gesetzlichen Krankenversicherung genommen, für die sie selbst finanziell aufkommen müssen, sodass Leistungsausschlüsse schnell verfassungsrechtliche Auswirkungen haben und Fragen nach der Vereinbarkeit insbesondere mit Grundrechten aufwerfen. 
Der Ausschluss nicht verschreibungspflichtiger Arzneimittel aus dem Leistungskatalog der gesetzlichen Krankenversicherung führt letztlich im Hinblick auf die Versicherten zu einem Eingriff in die allgemeine Handlungsfreiheit aufgrund der Einschränkung der Auswahl an Arzneimitteln sowie einem Eingriff in Art. 2 Abs. 1 GG i. V. m. dem Sozialstaatsprinzip. Darüber hinaus besteht eine Ungleichbehandlung von Versicherten, die mit nicht verschreibungspflichtigen Arzneimitteln versorgt werden und denen, die mit verschreibungspflichtigen Arzneimitteln versorgt werden müssen. Es liegt aber kein Eingriff in Art. 14 Abs. 1 GG vor, da schon krankenversicherungsrechtliche Ansprüche im Allgemeinen dem Eigentumsschutz nicht unterliegen und dies mithin ebenso für den Anspruch auf Versorgung mit Arzneimitteln gilt. Darüber hinaus kann keine Verletzung von der körperlichen Unversehrtheit durch den Ausschluss nicht verschreibungspflichtiger Arzneimittel geltend gemacht werden. Weder wird in die körperliche Unversehrtheit durch den Ausschluss nicht verschreibungspflichtiger Arzneimittel eingegriffen noch kann aus leistungsrechtlicher Perspektive des Grundrechts ein Anspruch begründet werden. Denn das Grundrecht auf körperliche Unversehrtheit gewährt keine Ansprüche auf bestimmte Leistungen oder ein Recht auf Gesundheit.

Der Ausschluss nicht verschreibungspflichtiger Arzneimittel ist schließlich mit dem Grundgesetz vereinbar. Grundrechte der gesetzlich Versicherten werden durch den Ausschluss nicht verletzt, da die Eingriffe in die allgemeine Handlungsfreiheit der Versicherten gerechtfertigt sind. Der Ausschluss ist aufgrund seiner Anknüpfung an Arzneimittel im unteren Preisbereich und der Ausnahmeregelungen in $\S 34$ Abs. 1 S. 2 und 5 SGB V zumutbar und insgesamt als verhältnismäßig zu erachten. Das Merkmal der Verschreibungspflicht ist zwar im Hinblick auf die Finanzierbarkeit der gesetzlichen Krankenversicherung nicht zielgenau, jedoch als sachgerechtes Kriterium anzusehen.

Auch wenn durch den Ausschluss nicht verschreibungspflichtiger Arzneimittel einzelne Gruppen von Versicherten verstärkt betroffen sind, so ist der Ausschluss ebenso hinsichtlich derer als verfassungsmäßig anzusehen. So ergeben sich etwa für chronisch kranke Versicherte keine anderen Erwägungen; eine Belastungsgrenze ist aus verfassungsrechtlicher Sicht nicht geboten. Des Weiteren kann zwar das medizinische Existenzminimum in Einzelfällen bei einkommensschwachen Versicherten verletzt sein, jedoch besteht in Ausnahmefällen die Möglichkeit über das SGB II oder SGB XII die Versorgung mit nicht verschreibungspflichtigen Arzneimitteln sicherzustellen. Hinsichtlich der besonderen Therapierichtungen ist die Regelung des $\S 12$ Abs. 6 AM-RL ausreichend, um der therapeutischen Vielfalt Rechnung zu tragen.

Bei den pharmazeutischen Herstellern stellen sich die Umsatzeinbußen, ebenso wie bei den Festbeträgen, als bloße Rechtsreflexe dar. Mit dem Ausschluss der OTC-Arzneimittel wird ein „ursprünglicher“" Wettbewerb, ohne gesetzliche Krankenversicherung, wiederhergestellt und dementsprechend ein freier Markt kreiert, auf dem die Arzneimittelhersteller miteinander konkurrieren und sich frei betätigen können. Die damit verbundenen Gewinnbeeinträchtigungen sind daher bloße Reflexe des wiederhergestellten Wettbewerbs. Darüber hinaus liegt auch keine Beeinträchtigung im Recht auf chancengleichen Marktzugang aus Art. 12 Abs. 1 GG i. V. m. Art. 3 Abs. 1 GG oder in der Eigentumsfreiheit vor. Aus europarechtlicher Sicht ist der Ausschluss mit der Warenverkehrsfreiheit grundsätzlich vereinbar. Ebenso ist sekundäres Unionsrecht in Form der Transparenzrichtlinie nicht verletzt. Diese findet nicht nur auf die OTC-Liste als Positivliste, sondern auch auf den Ausschluss selbst, der als Negativliste zu qualifizieren ist, Anwendung. Die Voraussetzungen, die die Transparenzrichtlinie an eine Negativliste stellt, nämlich das Bestehen einer Begründung, die auf objektiven und überprüfbaren Kriterien beruht, sowie deren Bekanntmachung, werden aber eingehalten. 


\section{Weitere Ausschlüsse nach § 34 SGB V}

\section{Der Ausschluss von Bagatellarzneimitteln - § 34 Abs. 1 S. 6 SGB V}

Der Ausschluss von Bagatellarzneimitteln schließt in den gesetzlichen genannten Fällen von Bagatellerkrankungen die Versorgung mit Arzneimitteln aus und überweist sie in die Eigenverantwortung der Versicherten. Ausgeschlossen sind nach dem Gesetz Arzneimittel zur Anwendung bei Erkältungskrankheiten und grippalen Infekten einschließlich der bei diesen Krankheiten anzuwendenden Schnupfenmittel, Schmerzmittel, hustendämpfenden und hustenlösenden Mittel, Mund- und Rachentherapeutika, wobei Arzneimittel zur Behandlung von Pilzinfektionen ausgenommen sind, sowie Abführmittel und Arzneimittel gegen Reisekrankheit. Es handelt sich dabei um einen Ausschluss nach Anwendungsgebieten, sodass die Versorgung mit Arzneimitteln nur in den vom Ausschluss umfassten Anwendungsgebieten erfolgt. Zudem ist der Ausschluss aufgrund teleologischer Auslegung auf Fälle von Bagatellerkrankungen beschränkt, sodass bei schweren Erkrankungen, wie sie bspw. in $\S 13$ Abs. 1 AM-RL genannt werden, der Ausschluss nicht greift.

Selbst wenn der Ausschluss nach $\S 34$ Abs. 1 S. 6 SGB V durch die Einführung des Ausschlusses nicht verschreibungspflichtiger Arzneimittel einen breiten Anwendungsbereich eingebüßt hat, ist er nicht als obsolet anzusehen. Denn im Bereich neuer Wirkstoffe, die der Verschreibungspflicht zunächst für drei Jahre unterfallen, ist die Regelung weiterhin anwendbar. Allerdings ist seither von einem schmalen Anwendungsbereich auszugehen. Des Weiteren ist der Ausschluss als verfassungsgemäß anzusehen, er verstößt insbesondere nicht gegen die Grundrechte der pharmazeutischen Unternehmer, und ist auch als europarechtskonform einzustufen.

\section{Der Ausschluss von Lifestyle-Arzneimitteln - $\S 34$ Abs. 1 S. 7-9 SGB V}

Die Regelung des $\S 34$ Abs. 1 S. 7-9 SGB V schließt sogenannte Lifestyle-Arzneimittel von der Verordnung zu Lasten der gesetzlichen Krankenversicherung aus. Dazu zählen gem. der nicht abschließenden Aufzählung des $\S 34$ Abs. 1 S. 8 SGB V solche Arzneimittel, die überwiegend zur Behandlung der erektilen Dysfunktion, der Anreizung sowie Steigerung der sexuellen Potenz, zur Raucherentwöhnung, zur Abmagerung oder zur Zügelung des Appetits, zur Regulierung des Körpergewichts oder zur Verbesserung des Haarwuchses dienen. Nach den Konkretisierungen des Gemeinsamen Bundesausschusses in $\S 14$ Abs. 1 AM-RL sind vom Ausschluss solche Arzneimittel umfasst, deren Einsatz im Wesentlichen durch die private Lebensführung bedingt ist oder die aufgrund ihrer Zweckbestimmung insbesondere (1) nicht oder nicht ausschließlich zur Behandlung von Krankheiten dienen, (2) zur individuellen Bedürfnisbefriedigung oder zur Aufwertung des Selbstwertgefühls dienen, (3) zur Behandlung von Befunden angewandt werden, die lediglich Folge natürlicher Alterungsprozesse sind und deren Behandlung medizinisch nicht notwendig ist oder (4) zur Anwendung bei kosmetischen Befunden angewandt werden, deren Behandlung in der Regel medizinisch nicht notwendig ist. Der gesetzliche Ausschluss von Arzneimitteln, die vorrangig der Erhöhung der Lebensqualität dienen, umfasst damit solche Arzneimittel, die nicht oder zumindest nicht primär der Behandlung einer Erkrankung, sondern dazu dienen, persönliche Wünsche der Versicherten zu erfüllen. Es handelt sich um Arzneimittel, die unabhängig vom Vorliegen einer Krankheit eingenommen werden können und bei denen der Wunsch des Versicherten nach Einnahme des Arzneimittels maßgeblich im Vordergrund steht. Entscheidend für den Ausschluss ist nicht die konkrete Anwendung im Einzelfall, sondern dass die Arzneimittel überwiegend in den vom Gesetz genannten Anwendungsgebieten und Therapiezielen eingesetzt werden. Sobald dies auf ein Arzneimittel zutrifft, gilt der Ausschluss umfassend, unabhängig davon, ob im konkreten Fall eine behandlungsbedürftige Krankheit vorliegt oder das Arzneimittel nur zur Verbesserung der individuellen Lebensqualität eingesetzt wird. 
Der Ausschluss ist als gesetzlicher Ausschluss konzipiert. Den Regelungen in der Arzneimittel-Richtlinie kommt allerdings grundsätzlich konkretisierende Wirkung zu, wobei die Aufnahme von Arzneimitteln in die Anlage II der Arzneimittel-Richtlinie, in der ausgeschlossene Lifestyle-Arzneimittel aufgelistet sind, grundsätzlich nur deklaratorische Bedeutung hat. Konstitutive Wirkung kommt der Aufnahme in die Anlage II jedoch dann zu, wenn der Gemeinsame Bundesausschuss, in Anlehnung an die gesetzlich genannten Fälle, weitere Arzneimittel ausgeschlossen hat.

Aus verfassungsrechtlicher Hinsicht verstößt der Ausschluss trotz seiner umfassenden Ausschlusswirkung nicht gegen die allgemeine Handlungsfreiheit oder das Grundrecht auf körperliche Unversehrtheit. Allerdings verstößt der generelle Ausschluss von Arzneimitteln zur Raucherentwöhnung gegen Art. 3 Abs. 1 GG. Denn dadurch wird die Tabak- oder Nikotinsucht gegenüber anderen Suchterkrankungen ungleich behandelt, da z. B. im Bereich der Alkoholentwöhnung Arzneimittel gewährt werden.

III. Der Ausschluss unwirtschaftlicher Arzneimittel - § 34 Abs. 3 SGB V

Der Ausschluss unwirtschaftlicher Arzneimittel nimmt gezielt einzelne Arzneimittel von der Verordnung aus, ohne sich auf bestimmte Indikationsgebiete oder Arzneimittelkategorien zu beziehen. Er ist aber sowohl im Hinblick auf Grundrechte der pharmazeutischen Unternehmer als auch im Hinblick auf Grundrechte der Versicherten verfassungsgemäß.

Es handelt sich dabei um keinen gesetzlichen Ausschluss, sondern um einen Ausschluss durch Arzneimittel-Richtlinien. § 34 Abs. 3 SGB V gibt dem Gemeinsamen Bundesausschuss aber keine Ausschlussermächtigung, sondern fingiert lediglich, dass Teile der Anlagen der Verordnung über unwirtschaftliche Arzneimittel in der gesetzlichen Krankenversicherung als Ausschlüsse des Gemeinsamen Bundesausschusses fortgelten sollen. Als Ausschlüsse kraft Arzneimittel-Richtlinie gelten laut der gesetzlichen Regelung nur die in Anlage 2 Nr. 2 bis 6 der Verordnung über unwirtschaftliche Arzneimittel aufgelisteten Arzneimittel und damit nur (ehemals) fiktiv zugelassene Arzneimittel. Für die Fortgeltung dieser Arzneimittelausschlüsse wurde nur die Rechtsform geändert, materiell-rechtliche Änderungen haben sich nicht ergeben. Zwar trifft die Arzneimittel-Richtlinie bisher noch keine Regelungen zur Fortgeltung und Änderung der Rechtsform von Teilen der ehemals durch Rechtsverordnung ausgeschlossenen unwirtschaftlichen Arzneimittel, jedoch legt die gesetzliche Regelung des $\S 34$ Abs. 3 S. 1 SGB V fest, dass die alten Rechtsverordnungsausschlüsse von Gesetzes wegen wie Richtlinienausschlüsse zu behandeln sind.

Die Arbeit erscheint im Verlag Duncker und Humblot, Berlin. 